\title{
Serum Complement C3 Level Is a Predictor of Preterm Birth in Preconception Care for Pregnancies With Systemic Lupus Erythematosus
}

\section{Yuri Hiramatsu}

Osaka Medical College

Kentaro Isoda

Osaka Medical College: Osaka Ika Daigaku

Takuya Kotani ( $\square$ in1242@osaka-med.ac.jp )

Osaka Medical College hospital https://orcid.org/0000-0003-2190-0780

\section{Eri Nakamura}

Osaka Medical College: Osaka Ika Daigaku

\section{Yumiko Wada}

Osaka Medical College: Osaka Ika Daigaku

\section{Youhei Fujiki}

Osaka Medical College: Osaka Ika Daigaku

\section{Shigeki Makino}

Osaka Medical College: Osaka Ika Daigaku

\section{Daisuke Fujita}

Osaka Medical College: Osaka Ika Daigaku

\section{Tohru Takeuchi}

Osaka Medical College: Osaka Ika Daigaku

\section{Research article}

Keywords: systemic lupus erythematosus, pregnancy, preconception care, preterm birth, serum complement 3 level

Posted Date: March 23rd, 2021

DOl: https://doi.org/10.21203/rs.3.rs-319630/v1

License: (c) (i) This work is licensed under a Creative Commons Attribution 4.0 International License. Read Full License 


\section{Abstract}

Background. This study aimed to clarify predictors of preterm birth in pregnancy of women with systemic lupus erythematosus (SLE). We investigated the predictors of preterm birth before pregnancy from the perspective of the importance of preconception care.

Methods. We analysed foetal outcomes of 108 pregnancies in 74 SLE patients. We compared prepregnancy clinical characteristics and disease activity in these women between the preterm birth and fullterm birth groups to select predictive factors for preterm birth before pregnancy.

Results. Eighty-three of 108 pregnancies resulted in live births, of which 27 (25.0\%) were preterm births. Pre-pregnancy serum complement 3 (C3) level was significantly lower in the preterm birth group (77.0 $\mathrm{mg} / \mathrm{dl})$ than the full-term birth group $(87.5 \mathrm{mg} / \mathrm{dl})(P=0.029)$. Multivariate analysis identified history of lupus nephritis (odds ratio: $5.734,95 \% \mathrm{Cl}: 1.568-21.010, \mathrm{P}=0.008)$ and low C3 level $(<85 \mathrm{mg} / \mathrm{dl})$ at prepregnancy (odds ratio: $4.498,95 \% \mathrm{Cl}: 1.296-15.616, \mathrm{P}=0.018$ ) as risk factors for preterm birth. The greater the number of these risk factors, the higher was the preterm birth rate $(P=0.0007)$. In the case of SLEDAl score $\leq 4$, the preterm birth rate was higher in the pre-pregnancy low C3 group ( $<85 \mathrm{mg} / \mathrm{dl}$ ) $(42.1 \%)$ than in the high $\mathrm{C} 3$ group $(\mathrm{C} 3 \geq 85 \mathrm{mg} / \mathrm{dl})(14.7 \%)(P=0.018)$.

Conclusion. Preconception care improves the outcome of SLE pregnancy. Especially for patients with a history of LN, treatment management focusing on pre-pregnancy serum complement titers is very important.

\section{Background}

Systemic lupus erythematosus (SLE) occurs primarily in women of reproductive age [1]. Recent improvements in treatment have dramatically improved the prognosis and quality of life of SLE patients, thus increasing their chances of becoming pregnant and giving birth. In addition, SLE patients are increasingly experiencing pregnancy and childbirth during the course of their treatment. Pregnant patients with SLE are at high risk of developing obstetric complications such as pre-eclampsia and eclampsia, as well as relapse of SLE disease activity during their pregnancy and puerperium.

Furthermore, pregnant patients with SLE have poor birth outcomes such as miscarriage, preterm birth, and foetal growth restriction, and there are many cases of preterm birth even if the pregnancy is successful [2-5]. Increased SLE disease activity during pregnancy, low serum levels of complement 3 (C3) or 4, high oral glucocorticoids (GC) dose, and high SLE disease activity have been reported as factors affecting preterm birth in pregnancy complicated by SLE [6,7]. Recently, the importance of preconception care has been advocated to reduce these risks that can adversely affect pregnancy outcomes.

Preconception care, which is planned management that prepares a women's body for future pregnancies from the perspective of improving the prognosis for the child, reducing the risk of complications, and maintaining maternal safety, is very important, especially for women with underlying illnesses $[3,8]$. However, there are few detailed reports about pre-pregnancy management in women with SLE, even 
though complications of lupus nephritis and high pre-pregnancy disease activity are known as predictors of preterm birth. Preconception care for pregnancies with SLE has not yet been established [6, 7]. We investigated the factors that influence preterm birth in pregnancies complicated by SLE using a retrospective case-control study method. We report on the predictors of preterm birth before pregnancy, especially in terms of the importance of preconception care.

\section{Materials And Methods}

Patients and study design

The subjects were pregnant SLE patients who were admitted to Osaka Medical College Hospital between January 2000 and October 2020. All of these patients fulfilled the 1997 American College of

Rheumatology revised criteria for SLE [9]. Rheumatologists and obstetricians assessed and managed the conditions of each mother and foetus once a month during pregnancy throughout the puerperal period.

To treat the patients and perform the present clinical study, past medical histories were investigated from the patients' previous medical records, and clinical and serological evaluations were performed.

As a systemic evaluation, serum C3 level and anti-dsDNA antibodies titres were evaluated every month. Antinuclear antibodies, anti-dsDNA antibodies, anti-RO/SSA and anti-RNP antibodies and antiphospholipid antibody (aPL) (i.e. against cardiolipin or b2-glycoproteins by ELISA or lupus anticoagulants) were also evaluated. For lupus nephritis, we determined that cases for which a definitive diagnosis could not be obtained by renal biopsy were nephritis based on the presence of proteinuria $(0.5$ $\mathrm{g} /$ day or higher) or cellular casts.

This study was conducted in accordance with the Declaration of Helsinki and its amendments, and was approved by the Osaka Medical College, Faculty of Medicine Ethics Committee (approval no. 1526). Written informed consent was obtained from each patient.

Treatment for SLE

We investigated GC and immunosuppressants such as azathioprine (AZA), tacrolimus (TAC), and hydroxychloroquine (HCQ) in regard to treatment contents before and during pregnancy. We also examined past use of GC pulse therapy and intravenous cyclophosphamide (IVCY). GC and immunosuppressants in use before pregnancy were continued during pregnancy. In the patients with SLE flare during pregnancy and postpartum, GC or immunosuppressants were newly administered and/or increased. Low-dose aspirin (LDA) was administered to patients positive for antiphospholipid antibody, and low-molecular-weight heparin (LMWH) was also used in addition to LDA in the patients with severe antiphospholipid antibody syndrome (APS), those who were positive twice or more for lupus anticoagulant, or those with a frequent history of obstetric complications.

Evaluation of foetal outcome and disease activity of SLE

Preterm birth was defined as delivery after 28 weeks but earlier than 37 weeks gestation. Spontaneous abortion was defined as completion of pregnancy earlier than 22 weeks gestation. Stillbirth was defined as foetal death at 28 weeks gestation or later. Abortion was performed at the patient's own request. We also evaluated the relapse and development of major organ lesions of SLE. In this study, based on the 
Asia Pacific League of Associations for Rheumatology LLDAS (lupus low disease activity state), the condition in which all of the following four items were satisfied was defined as 'SLE disease activity: less than low disease activity': (1) SLEDAI-2K (Systemic Lupus Erythematosus Disease Activity Index 2000) $\leq 4$, with no activity in major organ systems (renal, central nervous system, cardiopulmonary, vasculitis, and fever) and no haemolytic anaemia or gastrointestinal activity; (2) no new features of lupus disease activity compared with the previous assessment; (3) Physician Global Assessment (PhGA) (0-3) $\leq 1$; and (4) well-tolerated standard maintenance dosages of immunosuppressive drugs and approved biologics [10]. In this study, we excluded GC use from the disease activity endpoint as we are targeting pregnant patients in whom the combination of immunosuppressive drugs was restricted and the oral dose of GC was relatively high. Relapse of SLE disease activity was defined as an increase in the SLEDAI score $>3$. The activity of lupus nephritis was evaluated by proteinuria of $0.5 \mathrm{~g} /$ day or more, an increase in serum $\mathrm{Cr}$ level, and an increase in blood pressure.

Statistical analysis

Data are presented as the median value (interquartile range [IQR]) or number of subjects. Statistical analyses were performed with the chi-squared test or Fisher's exact test for binary data and with the Mann-Whitney U-test for continuous data between two groups. From these analyses, we selected candidates as the predictors of premature birth and then performed receiver operating characteristic (ROC) curve analysis to determine the most suitable cut-off level. A multivariate logistic regression model was used, and odds ratio (OR) and $95 \%$ confidence interval $(\mathrm{Cl})$ were calculated to examine independent risk factors for premature birth. A P-value of $<0.05$ was considered significant. Statistical calculations were performed using the statistical software JMP Pro for Windows, version 13.0 (SAS Institute Inc., Cary, NC, USA).

\section{Results}

Patient characteristics of pre-pregnancy

In total, 108 pregnancies in 74 patients were analysed, and the patients' characteristics are shown in Table 1. Of these 74 patients, 48 became pregnant once, 21 twice, three patients three times, one patient four times, and two patients became 5 times, respectively, during the study period. Patient age at pregnancy was $33(30-36)$ years. The SLEDAI score was $0(0-4)$. One patient was complicated with active lupus nephritis, but there were no active major organ lesions in the other patients. In the past, 29 patients $(26.9 \%)$ were diagnosed as having lupus nephritis. The serum C3 level before pregnancy was 81 (74-92) $\mathrm{mg} / \mathrm{dl}$. Anti-dsDNA antibody, anti-RO/SS-A antibody were positive in 73 (67.6\%) and 38 patients (35.2\%), respectively. Forty-six patients (43\%) were also positive for lupus anticoagulant activity, anticardiolipin IgG antibody, or anti- $\beta-2 \mathrm{GP} 1$ antibody. Ninety-one patients (84.3\%) were treated with $10(5-12)$ $\mathrm{mg} / \mathrm{dl}$ of GC before pregnancy. GC pulse therapy was previously administered in 27 patients $(25.0 \%)$. LDA was administered to $15 \mathrm{aPL}$-positive patients with a history of obstetrical abnormalities, and LMWH was co-administered from the first trimester. 


\section{Foetal outcome}

Table 1 shows the foetal outcomes of all 108 pregnancies. Eighty-three pregnancies (76.9\%) resulted in live births, 11 in miscarriage (at 5-11 weeks), 3 in stillbirth, and 11 in abortion. Two patients with APS experienced stillbirth at 22 and 25 weeks, respectively, and one patient had chromosome aberration at 15 weeks. The reasons for abortion were social background, poor control of the underlying disease, and taking medications that are contraindicated during pregnancy. The gestational age at delivery was 37.0 (33.5-37) weeks, and the foetal birth weight was 2760 (2289-3007) g. Twenty-seven (32.5\%) of the 82 live births were premature, and 2 were terminated before 30 weeks of gestation due to maternal preeclampsia. Lupus nephritis relapsed in two patients, and their pregnancies ended before 32 weeks.

Comparison of patient backgrounds between preterm and full-term birth groups

Table 2 shows the pre-pregnancy clinical backgrounds of the preterm and full-term birth groups. There was no difference in age, duration of illness, or SLEDAl score between the two groups. The preterm birth group had a significantly higher history of lupus nephritis than the full-term birth group $(P<0.001)$. There was no difference in the positive rate and antibody titre of anti-dsDNA antibody, or those in the other autoantibodies, between the two groups. Pre-pregnancy serum C3 levels were significantly lower in the preterm birth group $(77.5 \mathrm{mg} / \mathrm{dl})$ than in the full-term birth group $(87.5 \mathrm{mg} / \mathrm{dl})(P=0.027)$. In terms of treatment, there was no significant difference in the dose of GC administered before pregnancy, but the past use of GC pulse therapy was higher in the preterm birth group $(P=0.009)$.

Figure 1 shows the transition of each parameter before and during pregnancy between the full-term birth group and preterm birth group. In the preterm birth group versus full-term birth group, serum C3 levels were lower in the first, second, and third trimesters (81 vs $97 \mathrm{mg} / \mathrm{dl}, \mathrm{P}<0.001$; $88 \mathrm{vs} 107 \mathrm{mg} / \mathrm{dl}, \mathrm{P}<0.001$; and 100 vs $107 \mathrm{mg} / \mathrm{dl}, \mathrm{P}=0.024$, respectively). However, there was no significant difference between the two groups in anti-dsDNA antibody titre and SLEDAI score during pregnancy. The dose of GC during pregnancy was significantly higher in the preterm birth group in the second and third trimesters $(P=0.01$ and $P=0.001$, respectively). Forty-six patients

Selection of risk factors for preterm birth before pregnancy

We focused on the serum C3 level as a predictor of preterm birth before pregnancy based on a comparison of patient backgrounds in the preterm and full-term birth groups. We performed a ROC analysis to determine a cut-off value for pre-pregnancy serum C3 level that could indicate risks for preterm birth. As a result, the cut-off value for the serum C3 level was set to $84 \mathrm{mg} / \mathrm{dl}$ (sensitivity: 0.76, specificity: 0.54 , AUC: $0.653, P=0.043)$. We then performed a multivariate analysis to select risk factors for preterm birth before pregnancy. Candidate risk factors obtained from the results of univariate analysis comparisons between the two groups included age at pregnancy, which is commonly cited as a risk of preterm birth, history of lupus nephritis, past use of GC pulse therapy, and serum C3 level $<85 \mathrm{mg} / \mathrm{dl}$. The multivariate analysis using these variables identified a history of lupus nephritis (OR: $6.267,95 \% \mathrm{Cl}$ : 1.750 - 22.443, P = 0.005) and pre-pregnancy serum C3 level < 85 mg/dl (OR: 4.754, 95\% Cl: $1.373-16.461, \mathrm{P}=$ 0.014 ) as the risk factors for preterm birth (Table 3).

Figure 2 shows the relationship between the number of risk factors (a history of lupus nephritis and prepregnancy serum C3 level $<85 \mathrm{mg} / \mathrm{dl}$ ) and the number of weeks of delivery. The preterm birth rate 
(preterm birth/total birth ratio) was 7.7\% (2/26) in the patients without risk factors, 39.0\% (16/41) in those with 1 risk factor, and $66.7 \%$ (8/12) in those with 2 risk factors. The greater the number of these risk factors, the higher was the preterm birth rate $(P=0.0007)$.

Relationship between the pre-pregnancy serum C3 levels and the number of weeks of delivery in the cases of SLEDAI score $\leq 4$

In pregnancies with a SLEDAI score $>4,5$ of 7 (71.4\%) had preterm birth, and with a SLEDAI score $\leq 4,21$ of $72(29.1 \%)$ had preterm birth. Figure 3 shows the relationship between the pre-pregnancy serum C3 levels and the number of weeks of delivery in the cases of SLEDAI score $\leq 4$. The preterm birth rate was significantly higher in the pre-pregnancy low C3 group (<85 mg / dl) (42.1\%) than that in the high C3 group ( $\geq 85 \mathrm{mg} / \mathrm{dl})(14.7 \%)(P=0.018)$. The number of weeks of delivery was 37 (35-38) in the low C3 group, which was significantly earlier than that in the high C3 group $38(37-39)(P=0.044)$.

Maternal disease activity during pre-pregnancy and pregnancy

Among the 83 women delivering live babies, 7 (8.4\%) had SLE disease activity (medium disease activity or higher) before pregnancy. The pre-pregnancy serum C3 level was 81 (74-92) mg/dl of all. In addition, the presence of proteinuria or cellular casts of $0.5 \mathrm{~g}$ /day or more was observed before pregnancy in one woman.

Twenty women (24.0\%) had increased SLE disease activity (increase in the SLEDAI score of > 3) during pregnancy. Of the women who had undergone GC therapy before pregnancy, 43 (51.8\%) had an increased level of GC (8.9 mg/day). GC was newly introduced in 4 women (4.8\%) after their pregnancy was discovered. Due to SLE relapse during pregnancy, immunosuppressants were newly initiated during pregnancy in 5 women.

We compared SLE disease activity between the preterm and full-term birth groups (Figure 1). There was no significant difference in the SLEDAI score and anti-dsDNA antibody titres during either pre-pregnancy or pregnancy. Figure 4 shows a comparison of each parameter during pregnancy in the two groups [high ( $\geq 85 \mathrm{mg} / \mathrm{dl}$ ) and low C3 group (<85 mg/dl)] divided by serum C3 levels at pre-pregnancy. The serum C3 levels were significantly lower in the low C3 group than high C3 group during pre-pregnancy and pregnancy (pre-pregnancy: 95 vs 75 mg/dl, $\mathrm{P}<0.001$; first trimester: 103 vs 84 mg/dl, $\mathrm{P}<0.001$; second trimester: 114 vs $91 \mathrm{mg} / \mathrm{dl}, \mathrm{P}<0.001$; and third trimester: 113 vs 99 mg/dl, P < 0.001). The SLEDAl score was lower in the high C3 group before and in the first trimester than those in the low C3 group, but no significant correlation was observed in the anti-dsDNA antibody titre in either group during pre-pregnancy pregnancy.

\section{Discussion}

We investigated preterm birth predictors in a retrospective case-control study from the perspective of preconception care for pregnancies in women with SLE. The preterm birth group had lower serum C3 levels than the full-term birth group before pregnancy, which indicated that a pre-pregnancy serum C3 level $<85 \mathrm{mg} / \mathrm{dl}$ and a history of lupus nephritis are potential risk factors for preterm birth in pregnancies with SLE. We also found that the higher the number of these risk factors in pregnant patients with SLE, 
the higher was the preterm birth rate. Furthermore, it was clarified that the risk of preterm birth increases in cases without SLE disease activity (SLEDAI score $\leq 4)$, but with low C3 level $(<85 \mathrm{mg} / \mathrm{dl})$ before pregnancy. To our knowledge, there have been no previous reports showing a relation between prepregnancy serum C3 levels and preterm birth in pregnancies with SLE. This is the first report to show that the combination of pre-pregnancy serum C3 level and a history of lupus nephritis may predict preterm birth in pregnancies with SLE.

Pregnancy in women with SLE carries a higher risk of pregnancy complications than that in healthy individuals, and preterm births are reported to be 2.5 times higher [5]. Preterm birth is divided into spontaneous preterm birth due to premature rupture of the membrane and imminent preterm labour, and artificial preterm birth due to preeclampsia-induced hypertension and foetal adaptation. Preterm birth is the leading cause of neonatal death [11]. It is important to identify the risk factors of preterm births because in the surviving infants, the prevalence of diseases such as chronic lung disease, intraventricular haemorrhage, periventricular leukomalacia, and others is high, and they can affect the prognosis of child development [12]. There are some reports of outcomes in pregnancies with SLE, but many of the outcomes are discussed in relation to SLE disease activity and treatments during pregnancy. In a retrospective study of 267 SLE patients, high levels of anti-dsDNA antibodies and hypocomplementemia during the second trimester were associated with poor pregnancy outcomes such as foetal death and preterm birth in the pregnant patients with residual SLE disease activity [13]. Currently, there are limited reports on the management and treatment of SLE before pregnancy, which is important from the perspective of preconception care [4]. The present study showed for the first time that a pre-pregnancy serum C3 level $<85 \mathrm{mg} / \mathrm{dl}$, in addition to a history of lupus nephritis, is a risk factor for preterm birth. Furthermore, the result that the preterm birth rate increases in pregnancy with low serum C3 level $(<85$ $\mathrm{mg} / \mathrm{dl}$ ), even with SLEDAI $\leq 4$, suggests that pre-pregnancy C3 level may be more useful than SLEDAI for predicting preterm birth. The target value of a serum C3 level $>85 \mathrm{mg} / \mathrm{dl}$ is higher than the general treatment standard for non-pregnancy (serum C3 levels of 70-75 mg/dl) in SLE patients. This result shows that pregnancy is a special state in SLE that can exacerbate its condition, and it is necessary to keep the disease activity of pregnant SLE patients more stable than that in non-pregnant patients to avoid preterm birth. In addition, SLE patients with a history of lupus nephritis have an increased risk of preterm birth and may require strict control of disease activity based on serum C3 levels. The present study showed that in pregnancy with SLE, it is important to perform preconception care focusing on organ lesions such as lupus nephritis and serum C3 levels before pregnancy to improve the pregnancy outcome, even if there is no activity such as SLEDAI $\leq 4$.

Lupus nephritis and its history are associated with poor foetal outcomes and are also known as the risk of preterm birth in pregnancy with SLE [3]. This study also showed that a history of lupus nephritis is a risk of preterm birth. This result supports previous reports and indicates that we need to give special emphasis to preconception care for SLE patients with a history of lupus nephritis In pregnancy with SLE, it is necessary to pay attention to exacerbation of SLE disease activity and, at the same time, management of the mother. Pregnancy is known to trigger exacerbations of SLE [14]. So far, high disease activity during pregnancy, severe renal impairment, pulmonary hypertension, and central nervous system disorders have been reported as risk factors for SLE relapse [15]. The requirement for 
permitting pregnancy in SLE patients is generally low disease activity or remission during the 6 months prior to pregnancy [3]. Even in cases in which the disease activity of SLE is stable, SLE can worsen during pregnancy in one-fourth of women and cause preterm birth in one-third of them [4]. Furthermore, even in women with SLEDAI < 4 before pregnancy, the incidences of SLE flare and pregnancy complications are higher than those in healthy subjects [4,5]. The present study showed no difference in SLEDAl scores between the preterm and full-term birth groups from pre-pregnancy through the entire gestation period. However, the serum C3 levels in the preterm birth group were lower than those in the full-term birth group from before pregnancy through the entire pregnancy. Elevated serum complement levels are a phenomenon seen in normal pregnancies, but the pre-pregnancy rate of complement elevation was lower in the low C3 group ( $<85 \mathrm{mg} / \mathrm{dl}$ ) than that in the high C3 group ( $\geq 85 \mathrm{mg} / \mathrm{dl}$ ). In the present study, there were many women in whom the activity of SLE was well controlled, so this could be a possible reason for no difference in the SLEDAI score between the preterm and full-term birth groups. However, the serum C3 levels were different between the two groups, suggesting that changes in serum C3 levels may sharply reflect disease activity in pregnancies complicated by SLE.

This study has some limitations. First, it was performed with a small number of patients at a single institution. However, this enabled us to perform the study with unified criteria for monitoring the data of each patient. Second, the type of lupus nephritis could not be identified by renal biopsy in some patients, and therefore, evaluation of the outcome of pregnancy based upon disease type was not possible. Moreover, although the outcome of pregnancy and SLE disease activity in the mothers could be evaluated up to one year after delivery, subsequent growth of the children or long-term outcomes of the mothers could not be evaluated.

\section{Conclusion}

In preconception care of pregnancies in women with SLE, it is important to reduce the risk of obstetric complications such as preterm birth and improve the prognosis of the mothers and children by fully controlling disease activity and strict pregnancy planning. The present study showed that management focusing on the history of lupus nephritis in addition to serum C3 levels in preconception care is important for predicting and preventing preterm birth in women with SLE. Prediction of preterm birth stratified by these risk factors will help rheumatologists and obstetricians who are struggling to manage pregnancies with SLE. In addition, this preconception care may increase the safety of the life events of pregnancy and childbirth, which have been a challenge for SLE patients. We hope that further research will enable SLE patients to become pregnant, give birth safely, and enrich their lives by spreading preconception care to all pregnant women with SLE.

\section{Abbreviations}

SLE, systemic lupus erythematosus; C3, serum complement 3; SLEDAI, SLE Disease Activity Index; LLDAS, lupus low disease activity state; APS, antiphospholipid syndrome; GC, glucocorticoids ; IVCY, intravenous cyclophosphamide therapy; AZA, azathioprine; TAC, tacrolimus; HCQ, hydroxychloroquine; 
LDA, Low-dose aspirin; LMWH, low-molecular-weight heparin; anti-dsDNA Ab, anti-double-stranded DNA antibodies; anti-Ro/SS-Ab, anti-Ro/SSA antibodies; LA, lupus anticoagulant; aCL, anticardiolipin; B2Gp1, anti-B2-glycoprotein 1; anti-RNP Ab, anti-RNP antibodies; anti-Sm Ab, anti-Sm antibodies;

\section{Declarations}

\section{Ethics approval and consent to participate}

This study was approved by the ethical committee of Osaka Medical College (No. 1526) and complied with the guidelines of the Declaration of Helsinki. Written informed consent was obtained from each patient.

\section{Consent for publication}

Not applicable

\section{Availability of data and material}

The data underlying this article will be shared on reasonable request to the corresponding author.

\section{Competing interests}

The authors declare that they have no competing interests.

\section{Funding}

No specific funding was received from any funding bodies in the public, commercial or not-for-profit sectors to carry out the work described in this manuscript.

\section{Acknowledgements}

$\mathrm{YH}, \mathrm{KI}$, and TK designed the study; $\mathrm{YH}, \mathrm{KI}$ analysed the data; $\mathrm{YH}, \mathrm{KI}$, and TK wrote the manuscript; and TK and TT revised the manuscript. The authors thank EN, YW, YF, DH, and SM for their assistance in collecting data.

Disclosure statement: The authors declare no conflicts of interest.

\section{References}

1. Hardy CJ, Palmer BP, Morton SJ, et al. Pregnancy outcome and family size in systemic lupus erythematosus: a case-control study. Rheumatology. 1999;38:559-563.

2. Jill P Buyon, Mimi Y Kim, Marta M Guerra, et al. Predictors of Pregnancy Outcome in a Prospective, Multiethnic Cohort of Lupus Patients. Ann Intern Med. 2015;163(3):153-163. 
3. Andreoli L, Bertsias GK, Agmon-Levin N, et al. EULAR recommendations for women's health and the management of family planning, assisted reproduction, pregnancy and menopause in patients with systemic lupus erythematosus and/or antiphospholipid syndrome. Ann Rheum Dis. 2017;76:476485.

4. Y K Onno Teng, Edwin O W Bredewold, Ton J Rabelink, et al. An evidence-based approach to prepregnancy counselling for patients with systemic lupus erythematosus. Rheumatology 2018;57:1707-20.

5. Clowse ME, Jamison M, Myers E, James AH. A national study of the complications of lupus in pregnancy. Am J Obstet Gynecol. 2008;199:127:1-6.

6. Clowse ME, Magder LS, Petri M, et al. The clinical utility of measuring complement and anti-dsDNA antibodies during pregnancy in patients with systemic lupus erythematosus. J Rheumatol. 2011;38(6):1012-6

7. Clowse ME, Wallace DJ, Weisman M, et al. Predictors of preterm birth in patients with mild systemic lupus erythematosus. Ann Rheum Dis. 2013;72(9):1536-9

8. Lang AY, Boyle JA, Fitzgerald GL, et al. Optimizing preconception health in women of reproductive age. Minerva Ginecol. 2018;70(1):99-119.

9. M C Hochberg. Updating the American College of Rheumatology revised criteria for the classification of systemic lupus erythematosus. Arthritis Rheum 1997 Sep;40(9):1725

10. Kandane-Rathnayake R, Golder V, Louthrenoo W, et al. Development of the Asia Pacific Lupus Collaboration cohort. Int J Rheum Dis 2019;22(3):425-33.

11. Goldenberg RL, Culhane JF, lams JD, et al. Epidemiology and causes of preterm birth. Lancet 2008; 371(9606):75-84.

12. B Bohm, M Katz-Salamon. Cognitive development at 5.5 years of children with chronic lung disease of prematurity. Arch Dis Child Fetal Neonatal Ed 2003;88(2):101-5.

13. Clowse ME, Magder LS, Petri M, et al. The Clinical Utility of Measuring Complement and Anti-dsDNA Antibodies During Pregnancy in Patients with Systemic Lupus Erythematosus. J Rheumatol 2011;38(6):1012-6.

14. Smyth A, Oliveira GH, Lahr BD, et al. A systematic review and meta-analysis of pregnancy outcomes in patients with systemic lupus erythematosus and lupus nephritis. Clin J Am Soc Nephrol 2010;5:2060-8

15. Ponticelli C, Moroni G. Flares in lupus nephritis: incidence, impact on renal survival and management. Lupus 1998;7(9):635-8.

\section{Tables}


Table 1 Fetal outcome and patient characteristics and medications prior to pregnancy

$n=108$

Fetal outcome

Live birth, $\mathrm{n}(\%)$

$83(76.9)$

Full-term birth, $\mathrm{n}(\%)$

$56(51.9)$

Preterm birth, $\mathrm{n}(\%)$

$27(25.0)$

Miscarriage, $\mathrm{n}(\%)$

$11(10.2)$

Fetal loss, $\mathrm{n}(\%)$

$11(10.2)$

Elective abortion, $\mathrm{n}(\%)$

$3(2.8)$

Age, years old

$33(30-36)$

SLE duration, years

$8(4-13)$

History of lupus nephritis, n (\%)

$29(26.9)$

SLEDAI, score

$0(0-4)$

Past use of steroid pulse therapy, $n$ (\%)

27 (25.0)

Past use of IVCY, n (\%)

14 (13.0)

Prednisolone, $\mathrm{n}(\%)$

$91(84.3)$

Prednisolone dose, mg/day

$10(5-12)$

Azathioprine, n (\%)

23 (21.3)

Tacrolimus, $\mathrm{n}(\%)$

49 (45.3)

HCQ, n (\%)

11 (10.2)

Serum C3 level, mg/dL

$81(74-92)$

Positive Anti-ds-DNA Ab, n (\%)

$73(67.6)$

Anti-dsDNA Ab, IU/ml

$5.1(2.5-8.4)$

Positive anti-Ro/SS-A Ab, n (\%)

$38(35.2)$

Positive LA or anti-aCL-IgG Ab or anti- $\beta 2$ GP1 Ab

$46(43)$

Positive anti-RNP Ab, n (\%)

Positive anti-Sm Ab, n (\%)

9 (8.3)

Data are presented as median value (interquartile range) or number of patients.

Laboratory markers were evaluated in all patients. SLE: systemic lupus erythematosus; SLEDAI: SLE Disease Activity Index; IVCY: intravenous cyclophosphamide therapy; HCQ: hydroxychloroquine; C3: 
complement 3; anti-dsDNA Ab: anti-double-stranded DNA antibodies; anti-Ro/SS-Ab: anti-Ro/SSA antibodies; LA: lupus anticoagulant; aCL: anticardiolipin; B2Gp1: anti-B2-glycoprotein 1 
Table 2. Comparison of patient backgrounds of full-term birth and preterm birth

\begin{tabular}{|c|c|c|c|}
\hline & Full-term birth & Preterm birth & $\mathrm{p}$ value \\
\hline Number of patients & 56 & 27 & \\
\hline Gestational age at delivery, weeks & $38.0(37.0-39.0)$ & $34.0(32.0-36.0)$ & $<0.001$ \\
\hline Birth weight, g & $2892(2610-3115)$ & $2154(1568-2400)$ & $<0.001$ \\
\hline Age, years & $32(30-36)$ & $34(31-36)$ & 0.249 \\
\hline SLE duration, years & $7.5(3.3-11.8)$ & $9.0(5.0-16.0)$ & 0.056 \\
\hline Lupus nephritis, n (\%) & $8(14.3)$ & $14(51.9)$ & $<0.001$ \\
\hline APS, n (\%) & $21(37.5)$ & $12(44.4)$ & 0.634 \\
\hline SLEDAI, score & $0(0-4)$ & $1(0-4)$ & 0.225 \\
\hline SLEDAI $\geq 4, \mathrm{n}(\%)$ & $3(5.5)$ & $5(19.2)$ & 0.103 \\
\hline Past use of steroid pulse therapy, $n(\%)$ & $7(12.5)$ & $11(40.7)$ & 0.009 \\
\hline Past use of IVCY, n (\%) & $6(10.7)$ & $5(18.5)$ & 0.326 \\
\hline Prednisolone dose, mg/day & $9.0(3.1-11.0)$ & $10.0(7.0-12.0)$ & 0.181 \\
\hline Azathioprine, n (\%) & $14(25.0)$ & $3(11.1)$ & 0.245 \\
\hline Tacrolimus, n (\%) & $25(44.6)$ & $15(55.6)$ & 0.482 \\
\hline HCQ, n (\%) & $5(8.9)$ & $4(14.8)$ & 0.463 \\
\hline Serum C3 level, mg/dL & $87.5(75.0$ - 96.3) & $77.5(68.2-84.8)$ & 0.027 \\
\hline Positive Anti-ds-DNA Ab, n (\%) & $35(62.5)$ & $20(74.1)$ & 0.333 \\
\hline Anti-dsDNA Ab, IU/ml & $5.9(3.0-8.4)$ & $3.0(1.8-7.4)$ & 0.082 \\
\hline Positive anti-Ro/SS-A Ab, n (\%) & $19(33.9)$ & $10(37.0)$ & 0.810 \\
\hline Positive anti-RNP Ab, n (\%) & 19 (33.9) & $9(33.3)$ & 1.000 \\
\hline Positive anti-Sm Ab, n (\%) & $3(5.4)$ & $3(11.1)$ & 0.385 \\
\hline \multicolumn{4}{|c|}{$\begin{array}{l}\text { Data are presented as median value (interquartile range) or number of patients. The P-values were } \\
\text { estimated using Fisher's exact test or Wilcoxon rank sum test. }\end{array}$} \\
\hline \multicolumn{4}{|c|}{$\begin{array}{l}\text { SLE: systemic lupus erythematosus; SLEDAl: SLE Disease Activity Index; APS: antiphospholipid } \\
\text { syndrome; IVCY: intravenous cyclophosphamide therapy; HCQ: hydroxychloroquine; C3: complement } \\
\text { 3; Anti-dsDNA Ab: anti-double-stranded DNA antibodies; anti-Ro/SS-Ab: anti-Ro/SSA antibodies; anti- } \\
\text { RNP Ab: anti-RNP antibodies; anti-Sm Ab: anti-Sm antibodies. }\end{array}$} \\
\hline
\end{tabular}




\begin{tabular}{|llll|}
\hline \multicolumn{4}{|l|}{ Table 3. Risk factors of preterm birth (multivariate logistic regression model). } \\
\hline Age at pregnancy (per unit) & Odds ratio & $95 \% \mathrm{Cl}$ & p value \\
\hline History of lupus nephritis & 1.134 & $0.977-1.328$ & 0.086 \\
\hline Low serum complement 3 $(<85 \mathrm{IU} / \mathrm{ml})$ & 4.754 & $1.373-16.461$ & 0.014 \\
\hline Past use of steroid pulse therapy & 2.640 & $0.708-9.845$ & 0.148 \\
\hline
\end{tabular}

\section{Figures}
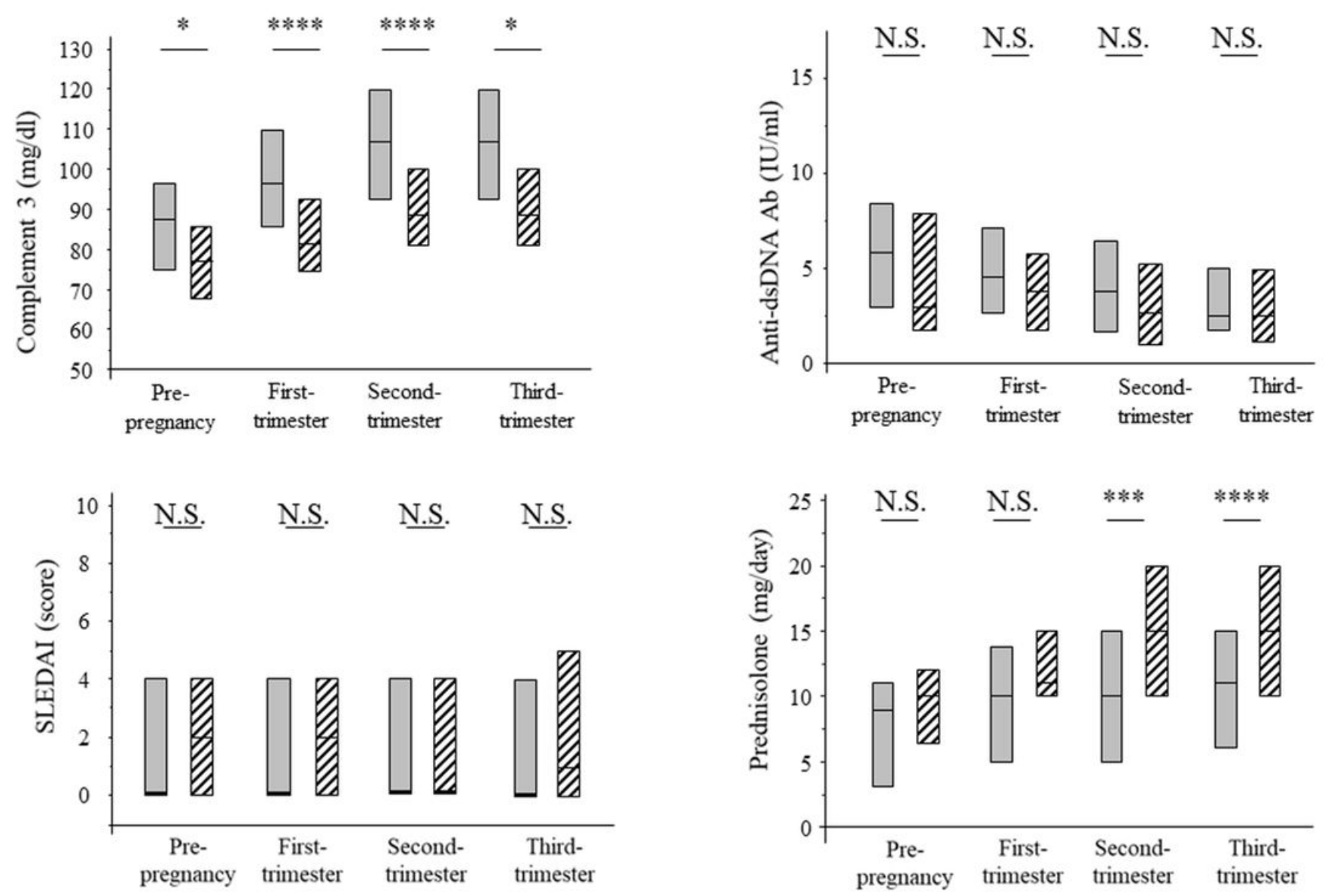

$\square$ Full term birth $\quad$ שIS Preterm birth

${ }^{*} \mathrm{p}<0.05,{ }^{* *} \mathrm{p}<0.01,{ }^{* * *} \mathrm{p}<0.005,{ }^{* * * *} \mathrm{p}<0.001$

\section{Figure 1}

The transition of each parameter before and after pregnancy between the full-term birth group and preterm birth group. A. Serum C3 levels of the preterm birth group were lower than those of the full-term 
birth group during all periods. B. There were no significant differences between in anti-dsDNA antibody titre during all periods. C. There were no significant differences in SLEDAl score during all periods. $D$. The doses of GC in the second and third trimesters were significantly higher in the preterm birth. ${ }^{*} P<0.05$, $\star \star \star P<0.005, * \star \star \star P<0.001$, N.S. Not significant

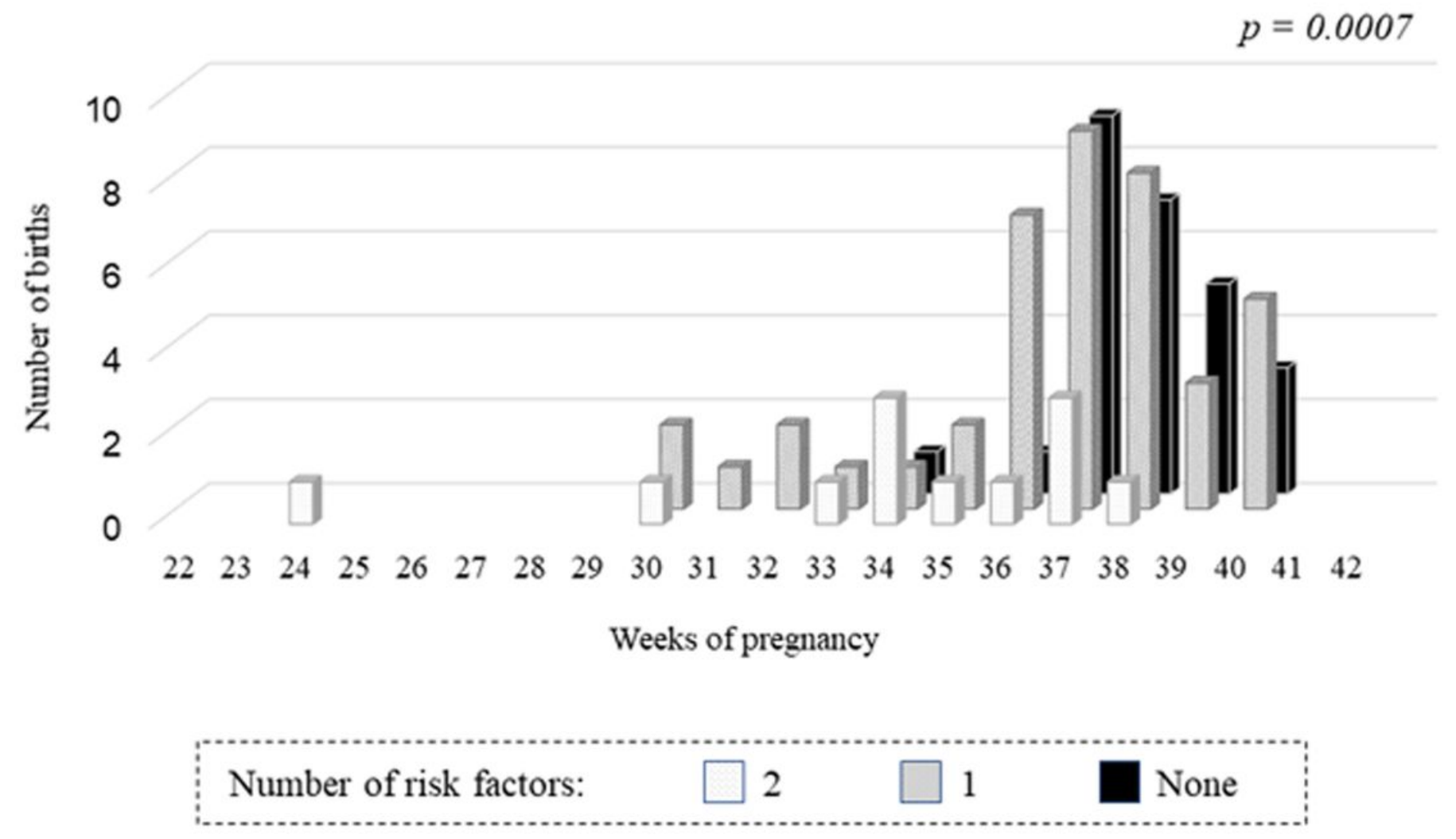

\section{Figure 2}

The relation between the number of risk factors and the number of weeks of delivery. The preterm birth rate (preterm birth/total birth ratio) was $7.7 \%(2 / 26)$ in the patients without risk factors (a history of lupus nephritis and pre-pregnancy serum C3 level $<85 \mathrm{mg} / \mathrm{dl}), 39.0 \%(16 / 41)$ in those with 1 risk factor, and $66.7 \%(8 / 12)$ in those with 2 risk factors. The greater the number of these risk factors, the higher was the preterm birth rate $(P=0.0007)$. 


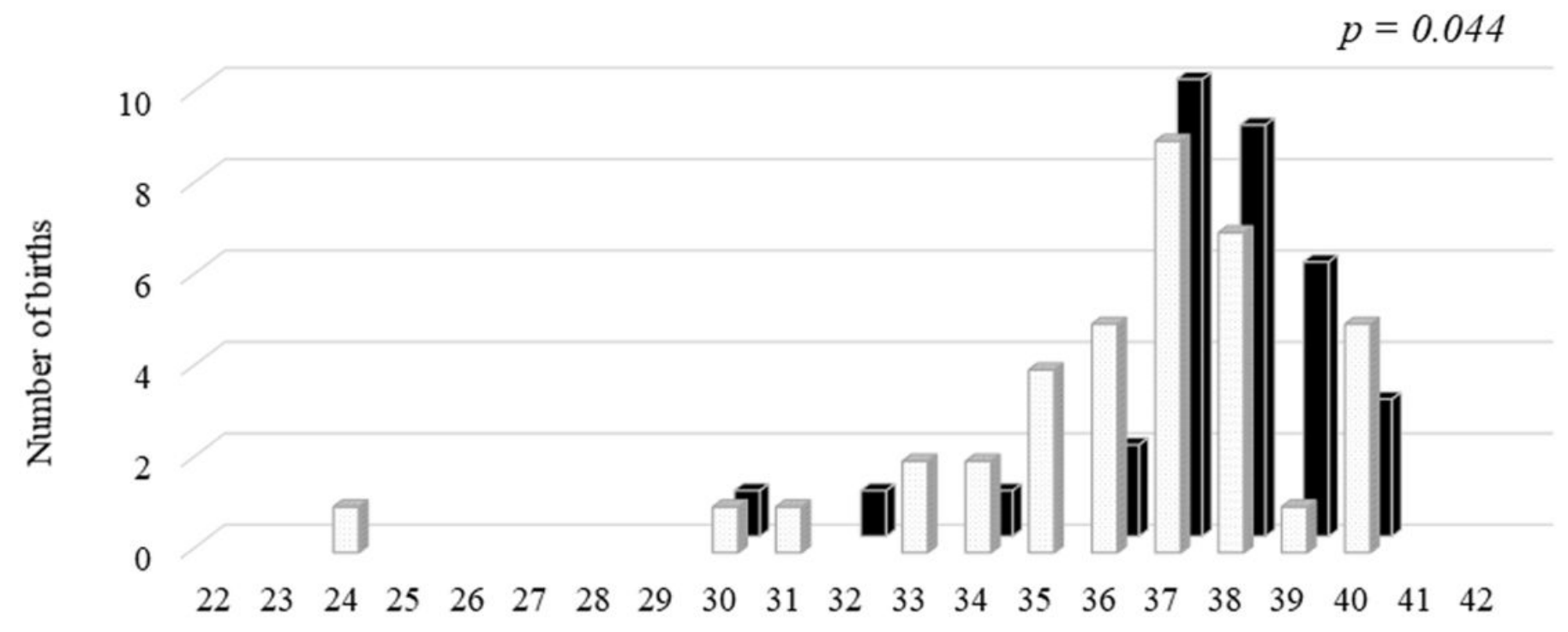

Weeks of pregnancy

Figure 3

The relationship between the pre-pregnancy serum C3 level and the number of weeks of delivery in the cases of SLEDAI score $\leq 4$. The preterm birth rate was significantly higher in the pre-pregnancy low C3 group ( $<85 \mathrm{mg} / \mathrm{dl})(42.1 \%)$ than that in the high C3 group (C3 $\geq 85 \mathrm{mg} / \mathrm{dl})(14.7 \%)(P=0.018)$. The number of weeks of delivery was 37 (35-38) in the low C3 group, which was significantly earlier than that in the high C3 group $38(37-39)(P=0.044)$. 

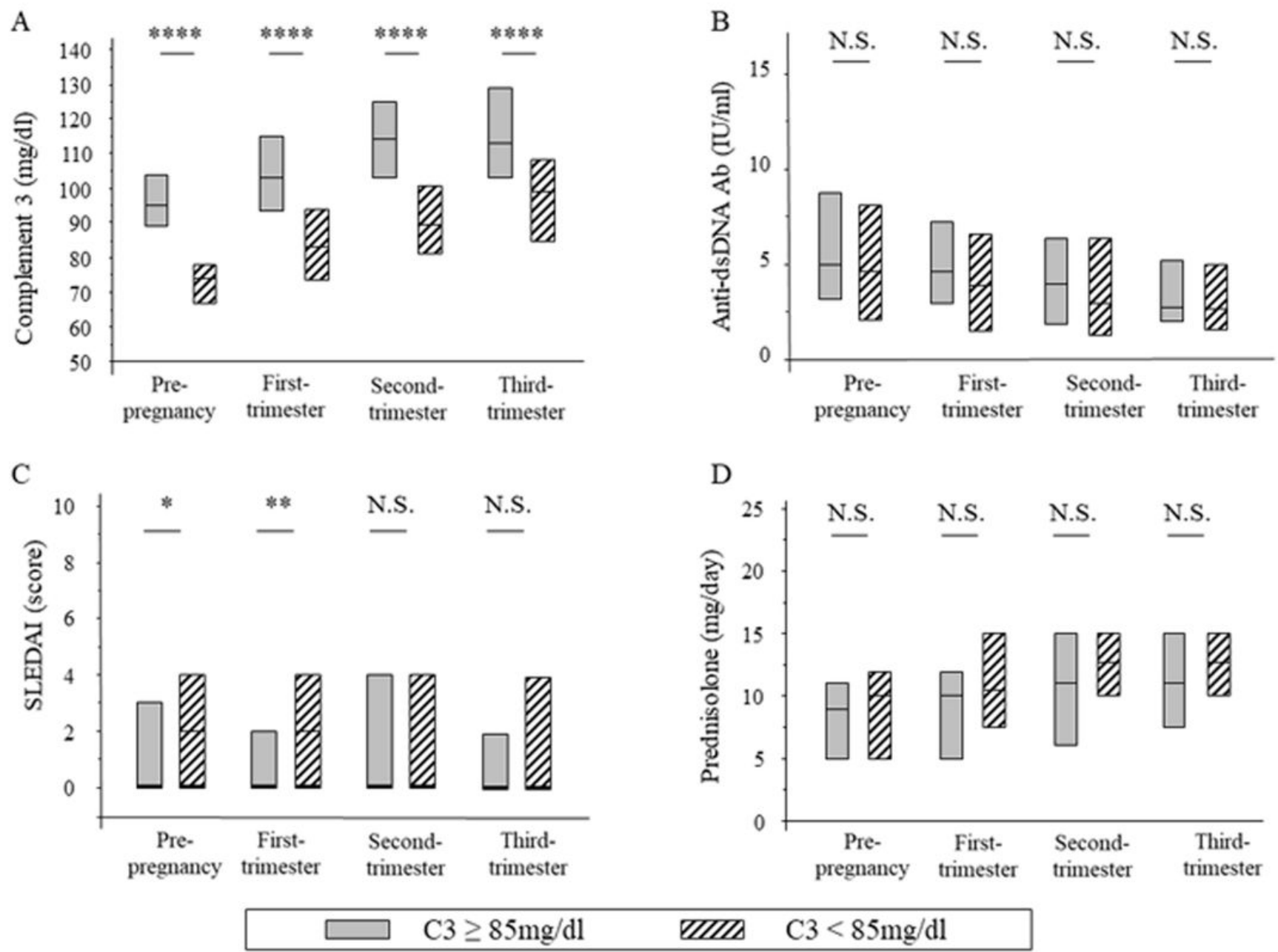

\section{Figure 4}

Comparison of each parameter during pregnancy between the high ( $\geq 85 \mathrm{mg} / \mathrm{dl}$ ) and low C3 group ( $<85$ $\mathrm{mg} / \mathrm{dl}$ ) at the time of pre-pregnancy. A. The serum C3 levels were lower in the low C3 group during all periods. B. The titres of anti-dsDNA antibodies were not significantly different during all periods. $\mathrm{C}$. SLEDAI scores at pre-pregnancy and the first trimester of pregnancy were lower in the high C3 group. D. The doses of glucocorticoids were not significantly different during all periods. ${ }^{*} P<0.05, * * P<0.01$, $\star \star \star * \mathrm{P}<0.001$, N.S. Not significant. 